\title{
Effect of haemodynamics on the risk of ischaemic stroke in patients with severe vertebral artery stenosis
}

To cite: Li Q, Zhou Y, Xing Y, et al. Effect of haemodynamics on the risk of ischaemic stroke in patients with severe vertebral artery stenosis. Stroke \& Vascular Neurology 2021;0. doi:10.1136/svn-2021-001283

- Additional supplemental material is published online only. To view, please visit the journal online (http://dx.doi.org/10. 1136/svn-2021-001283).

$\mathrm{QL}$ and $\mathrm{YZ}$ contributed equally.

Received 16 August 2021 Accepted 19 November 2021

D Check for updates

(C) Author(s) (or their employer(s)) 2021. Re-use permitted under CC BY-NC. No commercial re-use. See rights and permissions. Published by BMJ.

${ }^{1}$ Department of Vascular Ultrasonography, Xuanwu Hospital Capital Medical University, Beijing, China ${ }^{2}$ Beijing Diagnostic Center of Vascular Ultrasound, Xuanwu Hospital Capital Medical University, Beijing, China ${ }^{3}$ Center of Vascular Ultrasonography, Beijing Institute of Brain Disorders, Collaborative Innovation Center for Brain Disorders, Capital Medical University, Beijing, China

Correspondence to Dr Yang Hua; dryanghua99@163.com

Qing Li, ${ }^{1,2,3}$ Yinghua Zhou, ${ }^{1,2,3}$ Yingqi Xing, ${ }^{1,2,3}$ Jie Yang, ${ }^{1,2,3}$ Yang Hua (1) 1,2,3

\section{ABSTRACT}

Objectives Endovascular treatment strategies to optimise individualised care for patients with vertebral artery (VA) stenosis need to be revisited. This study aimed to investigate the relationship between net VA flow volume (NVAFV) and the risk of posterior circulation infarction (PCl) in a high-risk patient population.

Methods We screened 1239 patients with extracranial VA stenosis, of whom 321 patients with severe VA V1 segment stenosis $(\geq 70 \%)$ were enrolled in our study. We restratified the patients based on NVAFV and contralateral VA stenosis grades to analyse the proportion of each $\mathrm{PCl}$ mechanismlarge artery atherosclerosis and branch artery occlusive disease. Furthermore, we estimated the incidence of recurrent ischaemic stroke between groups with different NVAFV over a follow-up period of 2 years.

Results NVAFV was lower in the PCl group. Multiple logistic regression analysis showed that NVAFV is an independent risk factor for $\mathrm{PCl}$ and that the $\mathrm{OR}$ for $\mathrm{PCl}$ for the lowest NVAFV $(<112.8 \mathrm{~mL} / \mathrm{min})$ was 4.19 (1.76 to 9.95 , $\mathrm{p}=0.001$ ). In patients with severe carotid artery disease, the OR for the lowest NVAFV was 14.03 (3.18 to 61.92, $\mathrm{p}<0.001)$. The lower NVAFV group had a higher incidence of recurrent ischaemic stroke events than the higher NVAFV group (HR 2.978, 95\% Cls 1.414 to 6.272).

Conclusion Our study demonstrated that NVAFV, as estimated by colour duplex ultrasonography, was associated with the incidence of $\mathrm{PCl}$ and subsequent ischaemic events and that a high-risk population could be identified for further posterior circulation revascularisation.

\section{INTRODUCTION}

Approximately one-fifth of all ischaemic strokes occur in the posterior circulation system, and steno-occlusive disease of the vertebrobasilar arteries is an important underlying pathophysiological mechanism responsible for approximately one-quarter of these ischaemic stroke cases. ${ }^{1}$ Emerging data have suggested that vertebrobasilar stenosis predicts recurrent ischaemic events whose average annual incidence rate is $10 \%-15 \% .^{23}$ Therefore, effective secondary prevention strategies are important.

Endovascular treatment (EVT) of vertebral artery (VA) stenosis with the use of stents is a promising option and is widely implemented in clinical practice. ${ }^{45}$ However, both the Vertebral Artery Stenting Trial (VAST) ${ }^{6} 7$ and the Vertebral Artery Ischemia Stenting Trial (VIST) ${ }^{8}{ }^{9}$ have failed to demonstrate the superiority of stenting in preventing recurrent strokes in patients with symptomatic VA stenosis compared with optimal medical management. Based on data from the above trials, many experts have suggested that the current treatment strategies should be revised to provide optimal individualised care for all patients. Many issues need to be discussed before deciding to perform revascularisation, even in patients with severe VA stenosis. Considering the periprocedural risks associated with endovascular therapy, angioplasty and stenting are more likely to benefit specific groups of patients with the highest risk of ischaemic events.

It is now recognised that haemodynamic insufficiency plays an important role in the aetiology of stroke associated with VA stenosis. ${ }^{10}$ Colour duplex ultrasonography (CDU) is a dynamic real-time tool for the quantitative measurement of blood flow velocities and volumes. ${ }^{11-13}$ Given that EVT does not significantly benefit patients with symptomatic VA stenosis and the need for revising current treatment strategies for individualised patient care, this study aimed to investigate the relationship between net VA flow volume (NVAFV) and the risk of posterior circulation infarction (PCI) to find the high-risk patient population who could benefit from revascularisation.

\section{METHODS}

\section{Patients}

From January 2018 to January 2019, we enrolled patients who were diagnosed with severe VA V1 segment stenosis $(\geq 70 \%)$ based on vascular ultrasound findings that were further verified with CT angiography (CTA) or digital subtraction angiography (DSA). We retrieved additional data from patient history and laboratory tests during hospitalisation. Clinical information about age, sex, hypertension, diabetes mellitus, dyslipidaemia, 
smoking history, coronary heart disease history and medical history (including antiplatelet count and statin drug use) was collected from medical records. Laboratory test data for glucose, cholesterol, high-density lipoprotein and low-density lipoprotein levels were collected simultaneously. Two radiologists reviewed the MRI findings and located lesions, and more than two neurologists ascertained the presence of acute ischaemic stroke by combining clinical data and brain MRI findings. By conventional definition, transient ischaemic stroke (TIA) was defined as the occurrence of focal neurological symptoms and signs lasting for $<24$ hours. ${ }^{14}$ The presumed aetiology of stroke, which was deduced by the anatomic location and distribution of infarcts on brain MRI, was categorised into two types-large artery atherosclerosis (LAA) and branch artery occlusive disease $(\mathrm{Br} \mathrm{A})$-based on the independent determination of the two neurologists.

\section{Inclusion criteria}

(1) Diagnosis of severe unilateral or bilateral VA V1 segment stenosis $(\geq 70 \%)$, (2) vertebral blood flow volume quantification by CDU examination and (3) available clinical and laboratory data as well as MRI and CTA findings or DSA findings to confirm VA stenosis.

\section{Exclusion criteria}

(1) Definitive diagnosis of embolic infarction caused by atrial fibrillation or other cardiogenic diseases, (2) severe stenosis or occlusion of the intracranial vertebral or basilar arteries, (3) diagnosis of cerebral haemorrhage, encephalitis, posterior reversible encephalopathy syndrome, demyelinating disease or other non-vascular diseases and (4) diagnosis of vertebral dissection.

\section{Criteria for $70-99 \%$ extracranial VA stenosis}

CDU: according to the diagnostic criteria published previously by our research team, ${ }^{15}$ we defined $70 \%-99 \%$ stenosis of extracranial VA based on the following criteria: (1) peak systolic velocity (PSV) at the origin of the VA (PSV origin) $\geq 210 \mathrm{~cm} / \mathrm{s}$, (2) ratio of PSVorigin to the intervertebral VA segments $\geq 4.0$ and (3) end-diastolic velocity at the origin $\geq 50 \mathrm{~cm} / \mathrm{s}$. All three criteria had to be satisfied (figure 1)

CTA or DSA: according to the method of assessing carotid artery stenosis in the North American Symptomatic Carotid Endarterectomy Trial, ${ }^{16}$ the ratio between the residual luminal surface (inner-to-inner lumen) at the stenosis site and the surface of the distal normal lumen (inner-to-inner lumen), where there is no stenosis, was calculated. The degree of stenosis at the VA origin was stratified as $<50 \%, 50 \%-69 \%, 70 \%-99 \%$ and occlusion. We only enrolled the patients with unilateral or bilateral VA V1 segment stenosis ( $\geq 70 \%)$ (figure 1$)$.

\section{CDU quantification of blood flow volume in the VA}

Quantification of blood flow volume in the VA was performed by experienced sonographers with more than 5 years of experience using Philips EPIQ5 (Philips, Bothell, Washington) with $5.0-12.0-\mathrm{MHz}$ linear transducers. All the patients underwent CDU examination. We recorded the lumen diameter, PSV and end-diastolic velocity of each segment of the VA. The V2 segment (C3-C5 vertebrae intertransverse segments) of the VA was used to calculate the VA blood flow volume by multiplying the time-averaged mean velocity with the cross area of the segment. NVAFV was calculated as the sum of the bilateral VA blood flow volumes.

\section{PCI criteria}

PCI was diagnosed based on the following two criteria: (1) diffusion-weighted imaging (DWI) positive for infarcts in the brainstem, cerebellum thalamus, medial temporal lobe and occipital regions supplied by the VA, basilar artery (BA) and posterior cerebellar artery (PCA) and (2) DWI negative with low signals on T1-weighted images and high signals on T2-weighted and fluid-attenuated inversion recovery images simultaneously in the brainstem, cerebellum thalamus, medial temporal lobe and occipital regions supplied by the VA, BA and PCA.

We categorised PCI mechanisms according to the classification criteria proposed by Caplan et $a l^{10} 17$ and the TOAST (Trial of ORG 10172 in acute stroke treatment) classification ${ }^{18}$ as follows: (1) large artery occlusive disease haemodynamic mechanism (LAH), with MRI showing an ischaemic lesion located in the occlusion territory that is larger than a single-branch artery territory or that extends in distal fields, (2) $\mathrm{Br} \mathrm{A}$, with MRI showing infarcts limited to a single penetrating or circumferential branch territory and (3) artery-to-artery embolism (EmbA-A), with MRI showing multiple scattered infarcts in the territory of the main intracranial artery or the superficial branches in the posterior circulation area. We grouped LAH and EmbA-A into the LAA group (figure 2).

\section{Follow-up recurrent ischaemic events}

After initial hospitalisation for an ischaemic event, we assessed the patients for subsequent ischaemic events over 2 years of follow-up. We defined an ischaemic stroke as recurrent only when a new clinical symptom was accompanied by a new lesion detected on MRI. The endpoint time was determined as the time for the first recurrent ischaemic event to occur.

\section{Statistical analysis}

Normally distributed continuous variables were expressed as mean $\pm \mathrm{SD}$. Continuous variables with skewed distribution were expressed as medians (IQRs) and categorical variables were expressed as numbers (percentages). For equivalent variables with a normal distribution, independent Student's t test was used to compare the PCI and non-PCI groups. The Mann-Whitney U test was used to compare categorical variables and abnormally distributed variables between these two groups. Receiver operating characteristic (ROC) analysis was performed to identify the cut-off value. Area under the curve, optimal threshold, sensitivity and specificity were calculated. Logistic regression analysis was performed to screen for independent 

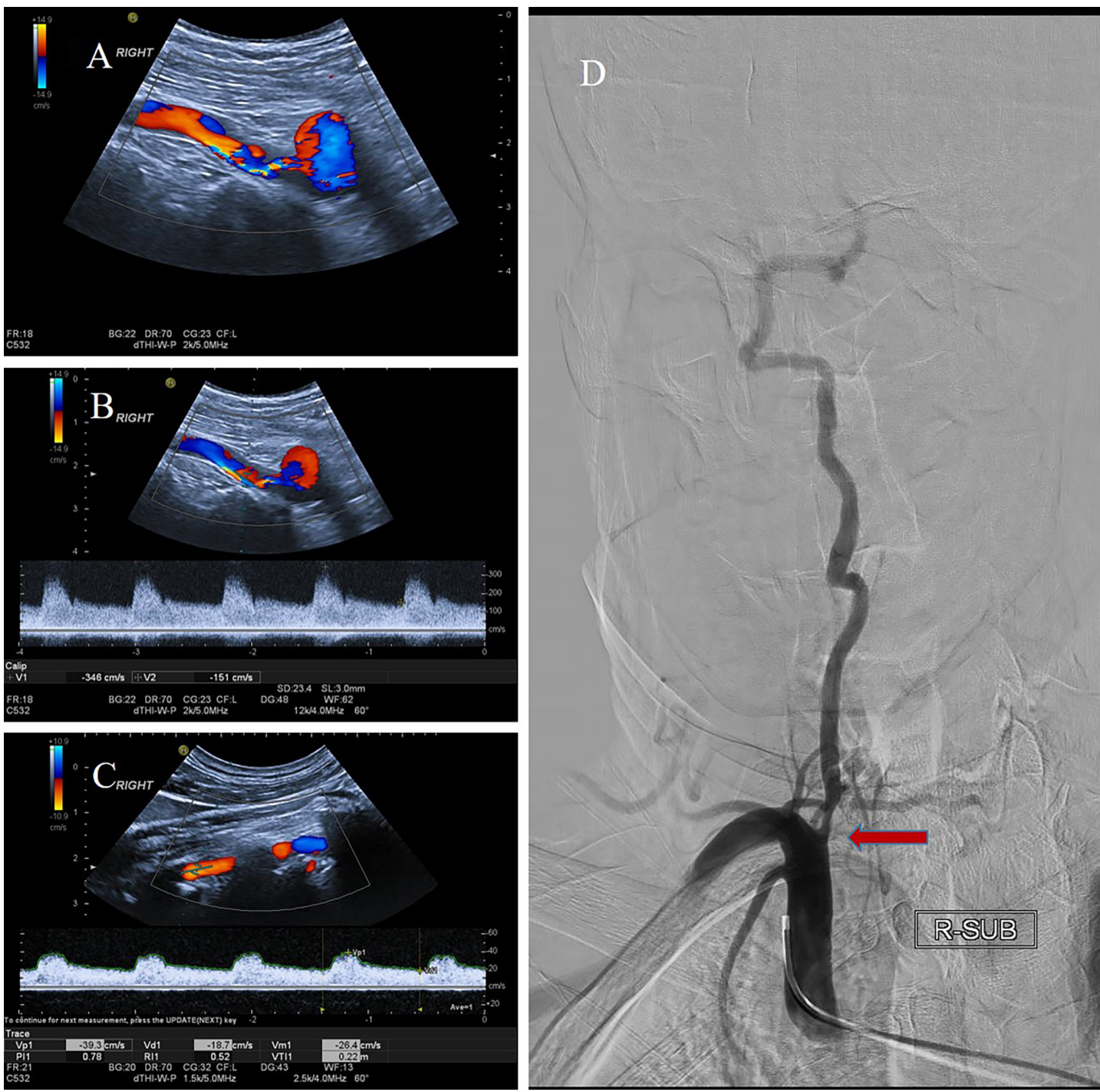

Figure 1 Patients with severe stenosis at V1 segment of right vertebral artery (RVA). (A) Colour Doppler image shows severe stenosis at V1 segment of RVA. (B) Spectral Doppler waveform shows high-velocity flow in V1 segment of RVA. (Peak systolic velocity: $346 \mathrm{~cm} / \mathrm{s}$; end-diastolic velocity: $151 \mathrm{~cm} / \mathrm{s}$ ). (C) Spectral Doppler waveform shows stable flow in C3-C4 intervertebral segment of RVA. (Peak systolic velocity:39 cm/s; end-diastolic velocity:18 cm/s). (D) Digital subtraction angiogram shows severe stenosis at V1 segment of RVA.

risk factors after adjusting for all confounders, including traditional factors and parameters distributed differently in each group. Statistical significance was defined as a twosided $p$ value of $<0.05$. CIs were set at $95 \%$. Kaplan-Meier analysis was used to compare the incidence of recurrent ischaemic events for groups with different NVAFV. Statistical analyses were performed using the IBM SPSS (V.22.0) and GraphPad Prism (V.6.01) software.

\section{RESULTS}

A total of 1239 patients were diagnosed with extracranial VA stenosis at our department, and 822 patients were excluded because of extracranial non-severe VA stenosis or bilateral VA occlusion; another 86 patients were excluded because of severe intracranial vertebral or BA stenosis. The remaining 331 successive patients were reviewed, and 10 patients with no MRI findings were excluded. Finally, 321 participants with complete clinical information and CDU data were enrolled in our study. After 2 years, 262 (81.6\%) patients completed follow-up. PCI was observed in $142(44.2 \%)$ of the 321 patients, of which $94(66.2 \%)$ were categorised into the LAA group and 48 (33.8\%) into the Br A group; 54 (16.8\%) patients with ischaemic symptoms were diagnosed with TIA due to no abnormal MRI signals in the posterior circulation area. Furthermore, 125 patients had either asymptomatic or anterior circulation infarction and were categorised into the non-PCI group.

\section{Patient demographic characteristics and blood flow volume} Age and sex distributions were similar between the groups. The prevalence of hypertension, diabetes mellitus, coronary heart disease, dyslipidaemia, smoking history and medical history was not significantly different between 

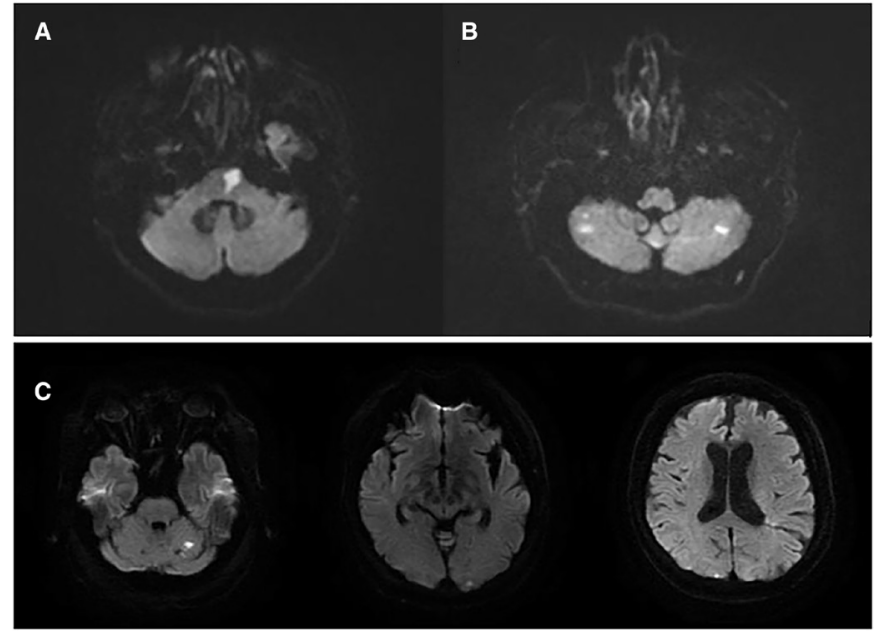

Figure $2 \mathrm{PCl}$ mechanisms in patients with symptomatic vertebral artery stenosis. (A) A single lesion in the penetrating artery territory indicating $\mathrm{Br} \mathrm{A}$. (B) Multiple lesions in posterior circulation territories indicate LAH (large artery occlusive disease haemodynamic mechanism). (C) Multiple cortical infarctions indicating EmbA-A. Br A, branch artery occlusive disease; EmbA-A, artery-to-artery embolism; $\mathrm{PCl}$, posterior circulation infarction.

the groups; the same was noted for glucose, cholesterol, high-density lipoprotein and low-density lipoprotein levels. The proportion of severe anterior circulation artery stenosis was higher in the non-PCI group than in the PCI group $(62.4 \%$ vs $31.7 \%, \mathrm{p}<0.001)$. Patients with severe contralateral VA stenosis were more likely to experience PCI $(32.4 \%$ vs $8.8 \%, \mathrm{p}<0.001)$. Both NVAFV and the flow volume in the severely stenotic side were lower in the PCI group than in the non-PCI group $(76.4 \pm 46.6$ vs $64.5 \pm 40.4, \mathrm{p}=0.027 ; 182.8 \pm 70.2$ vs $141.0 \pm 60.9, \mathrm{p}<0.001$, respectively), and this was also the case in the PCI +TIA group $(76.4 \pm 46.6$ vs $66.8 \pm 41.3, \mathrm{p}=0.056 ; 182.8 \pm 70.2$ vs $147.3 \pm 61.2, \mathrm{p}<0.001$, respectively). The detailed information is provided in table 1 .

\section{Patient demographics in groups with different NVAFV}

For classification, NVAFV was equally divided into four parts with values ranging from low to high. The clinical examination values and basic laboratory data were not significantly different among the groups with different NVAFV. The proportion of PCI was much higher in the lower NVAFV (L-NVAFV) group than in the higher NVAFV (H-NVAFV) group (65\% vs $49.4 \%$ vs $36.2 \%$ vs $26.2 \%$, $\mathrm{p}<0.001)$. This result was consistent in the PCI +TIA group ( $80 \%$ vs $60.5 \%$ vs $62.5 \%$ vs $41.2 \%$, p $<0.001)$. The mechanism of PCI exhibited a remarkably different distribution among the different NVAFV groups, and the prevalence of LAA was inversely correlated to the increase in NVAFV (51.2\% vs $30.9 \%$ vs $23.8 \%$ vs $11.2 \%, \mathrm{p}<0.001)$, whereas the prevalence of $\mathrm{Br} \mathrm{A}$ was similar among these groups. NVAFV had increased in patients with severe anterior circulation artery stenosis. Patients with $>70 \%$ contralateral VA stenosis accounted for $45 \%$ of patients in the L-NVAFV group and only $7.5 \%$ in the H-NVAFV group. A detailed description of this is provided in online supplemental table 1.

\section{Multiple logistic regression analysis of PCI and NVAFV}

As shown in table 2, NVAFV was a significant predicting factor for PCI or PCI+TIA after adjusting for age, sex, contralateral VA stenosis and anterior severe stenosis. Compared with the highest NVAFV (>199.1 mL/min), the lowest NVAFV $(<112.8 \mathrm{~mL} / \mathrm{min})$ had a profound effect on the prevalence of PCI and PCI+TIA (OR 4.19, $95 \%$ CI 1.76 to $9.95, \mathrm{p}=0.001$; OR $3.63,95 \%$ CI 1.63 to $8.10, \mathrm{p}=0.001)$. Contralateral VA stenosis of $<70 \%$ had an effect on the prevalence of PCI or PCI+TIA, similar to VA hypoplasia; however, contralateral VA stenosis of $>70 \%$ or occlusion had a higher tendency to result in PCI or PCI + TIA compared with hypoplasia and contralateral VA stenosis of $<70 \%$ (OR 3.32, 95\% CI 0.96 to 11.46, $\mathrm{p}=0.058$; OR $3.23,95 \%$ CI 0.98 to $10.65, \mathrm{p}=0.055)$. We separated these patients into two groups based on the presence of severe stenosis $(\geq 70 \%)$ in the anterior circulation artery. The lowest NVAFV had a predominant effect on the prevalence of PCI compared with the highest NVAFV in patients with severe stenosis of the anterior circulation arteries (OR 14.03, 95\% CI 3.18 to 61.92, $\mathrm{p}<0.001$ ). Contralateral VA stenosis of $>70 \%$ or occlusion tended to induce PCI or PCI +TIA compared with contralateral VA hypoplasia or stenosis of $<70 \%$ in patients without severe anterior circulation artery stenosis (OR 6.67, 95\% CI 0.91 to $49.04, \mathrm{p}=0.062$; OR $6.80,95 \% \mathrm{CI} 0.98$ to 47.13 , $\mathrm{p}=0.052$ ); however, this relationship was not found in patients with severe anterior circulation artery stenosis.

\section{ROC analysis of NVAFV for PCI}

The ROC curve analysis identified an NVAFV cut-off value of 168.21 as a threshold to identify patients at high risk for PCI (sensitivity 0.725 , specificity 0.576 , area under curve 0.683 , Youden's index 0.301).

\section{Proportion of PCI under the combined effect of different bilateral VA flow volumes and contralateral VA stenosis} grades

We divided the patients into four groups according to the NVAFVs separated by the threshold of 168.21 and contralateral VA stenosis grades to determine PCI distribution. Group 1 was defined as contralateral VA nonsevere stenosis (CVAS $(-))$ and H-NVAFV. Group 2 was defined as contralateral VA severe stenosis (CVAS $(+))$ and H-NVAFV. Group 3 was defined as CVAS(-) and L-NVAFV. Group 4 was defined as CVAS $(+)$ and L-NVAFV. The proportion of PCI gradually increased among the four groups. Group 1 had the lowest proportion of PCI $(25.4 \%)$, whereas PCI was more frequently observed in group $4(86.0 \%)$. In addition, group 3 had a higher prevalence of PCI than group 2 (56.6\% vs $42.9 \%$ ) (figure 3 ).

Percentile of different pathological mechanisms of PCI in the four groups

LAA was the most frequent pathological mechanism of PCI in group 4 and the least frequent in group $1(69.1 \%$ 
Table 1 Patient demographic characteristics and flow volume in each group

\begin{tabular}{|c|c|c|c|c|c|}
\hline & No-PCI group (125) & $\mathrm{PCl}$ group (142) & $P$ value & PCI+TIA (196) & $P$ value \\
\hline Age & $65.2 \pm 8.7$ & $65.5 \pm 9.2$ & 0.760 & $65.4 \pm 8.9$ & 0.912 \\
\hline Sex (male) & $105(84.0)$ & $131(90.8)$ & 0.090 & $169(86.2)$ & 0.583 \\
\hline Hypertension & $100(80.0)$ & $107(75.4)$ & 0.364 & $149(76.0)$ & 0.405 \\
\hline Coronary heart disease & $28(22.4)$ & 27 (19.0) & 0.495 & $44(22.4)$ & 0.992 \\
\hline Diabetes mellitus & $51(40.8)$ & $52(36.6)$ & 0.484 & $66(33.7)$ & 0.196 \\
\hline Smoking history & $82(65.6)$ & 97 (68.3) & 0.638 & $127(64.8)$ & 0.883 \\
\hline Dyslipidaemia & $58(46.4)$ & $65(45.8)$ & 0.919 & $91(46.4)$ & 0.996 \\
\hline Glucose (mmol/L) & $6.1 \pm 2.3$ & $5.8 \pm 1.7$ & 0.192 & $5.7 \pm 1.7$ & 0.106 \\
\hline Cholesterol (mmol/L) & $3.7 \pm 0.9$ & $3.5 \pm 0.9$ & 0.109 & $3.5 \pm 0.9$ & 0.213 \\
\hline $\mathrm{HDL}$ (mmol/L) & $2.1 \pm 0.7$ & $2.0 \pm 0.7$ & 0.258 & $2.1 \pm 0.7$ & 0.282 \\
\hline LDL (mmol/L) & $2.1 \pm 0.8$ & $2.0 \pm 0.8$ & 0.258 & $2.1 \pm 0.7$ & 0.282 \\
\hline Antiplatelet & $75(60)$ & $91(64.1)$ & 0.492 & $123(62.8)$ & 0.621 \\
\hline Statin & $76(60.8)$ & $86(60.6)$ & 0.968 & $117(59.7)$ & 0.844 \\
\hline Mechanism & & & $<0.001$ & & $<0.001$ \\
\hline LAA & 0 & $94(66.2)$ & & $94(48.0)$ & \\
\hline $\mathrm{Br} \mathrm{A}$ & 0 & $48(33.8)$ & & $48(24.5)$ & \\
\hline Combined with severe anterior artery stenosis & $78(62.4)$ & $45(31.7)$ & $<0.001$ & $56(28.6)$ & $<0.001$ \\
\hline Contralateral VA stenosis & & & $<0.001$ & & $<0.001$ \\
\hline$<70 \%$ & $107(85.6)$ & $88(60.6)$ & & $127(64.8)$ & \\
\hline$\geq 70 \%$ or occlusion & $11(8.8)$ & $46(32.4)$ & & $56(28.6)$ & \\
\hline Hypoplasia (diameter less than $2.5 \mathrm{~mm}$ ) & $7(5.6)$ & $10(7.0)$ & & $13(6.6)$ & \\
\hline V2 segment diameter(mm) & $3.3 \pm 0.6$ & $3.4 \pm 0.6$ & 0.206 & $3.4 \pm 0.6$ & 0.334 \\
\hline Ipsilateral VA flow volume(ml/min) & $76.4 \pm 46.6$ & $64.5 \pm 40.4$ & 0.027 & $66.8 \pm 41.3$ & 0.056 \\
\hline NVAFV (mL/min) & $182.8 \pm 70.2$ & $141.0 \pm 60.9$ & $<0.001$ & $147.3 \pm 61.2$ & $<0.001$ \\
\hline
\end{tabular}

Severe anterior artery stenosis: unilateral or bilateral extracranial carotid artery severe stenosis $(\geq 70 \%)$ or occlusion.

$\mathrm{Br} \mathrm{A}$, branch artery occlusive disease; HDL, high density lipoprotein; LAA, large artery atherosclerosis; LDL, low density lipoprotein; NVAFV, net vertebral artery flow volume; PCI, posterior circulation infarction; TIA, transient ischaemic stroke; VA, vertebral artery.

vs $8.3 \%$ ). The pathological mechanism Br A for PCI was equally distributed among the four groups. LAA was more prevalent in group 3 than in group 2 (28.1\% vs $15.1 \%)$. Figure 4 provides detailed information about these four groups in this regard.

\section{Follow-up recurrent ischaemic events in the L-NVAFV and H-NVAFV groups}

A total of $262(81.6 \%)$ patients completed the 2-year follow-up. On comparing the 2-year recurrent ischaemic stroke incidence between the lower and H-NVAFV groups, the lower NVAFV group showed a higher incidence of stroke (HR 2.978, 95\% CI 1.414 to 6.272, $\mathrm{p}=0.0125$; figure 5, online supplemental table 2 than the H-NVAFV group.

\section{DISCUSSION}

Our study demonstrated that NVAFV, which is calculated using extracranial VA ultrasound, may represent an effective means to screen high-risk patients as a target population for future endovascular intervention. NVAFV was associated with non-cardioembolic PCI in patients with severe unilateral or bilateral VA stenosis, especially via the LAA mechanism. In patients with different contralateral VA stenosis grades, NVAFV exhibited a superior effect, representing the adequacy of the posterior circulation.

Previous research has discovered that the normal range of NVAFV is $102.4-301.0 \mathrm{~mL} / \mathrm{min}$ (fifth-95th percentile) and that NVAFV of $<100 \mathrm{~mL} / \mathrm{min}$ is considered abnormal. ${ }^{11}$ The odds of non-cardioembolic PCI with NVAFV of $<90$ and $100 \mathrm{~mL} / \mathrm{min}$ were reported to be 3.26 and 3.48, respectively, in Lee et al.'s study. ${ }^{13}$ In our study, the prevalence of PCI or PCI +TIA was higher in the lowest NVAFV group than in the highest NVAFV group, which is consistent with the findings of a previous study.

According to the comprehensive overview by Lattanzi $e t$ $a l^{19}$ of four available randomised clinical trials, including Carotid and Vertebral Artery Transluminal Angioplasty Study, VAST, VIST and Stenting and Aggressive Medical Management for Preventing Recurrent stroke in Intracranial Stenosis, which focus on comparing EVT plus medical treatment and medical treatment alone for patients with symptomatic intracranial and extracranial vertebrobasilar system stenosis, no superiority effect of 
Table 2 Multiple logistic regression analysis of $\mathrm{PCl}$ and NVAFV

\begin{tabular}{|c|c|c|c|c|}
\hline & $\mathrm{PCl}$ & & $\mathrm{PCl}+\mathrm{TIA}$ & \\
\hline \multicolumn{5}{|l|}{ NVAFV } \\
\hline 199.1-highest* & Ref & Ref & Ref & Ref \\
\hline $0-112.8$ & 4.19 (1.76 to 9.95$)$ & 0.001 & $3.63(1.63-8.10)$ & 0.002 \\
\hline $112.9-157.1$ & 1.97 (0.93 to 4.16$)$ & 0.077 & $1.60(0.81-3.17)$ & 0.179 \\
\hline 157.2-199 & $1.73(0.80$ to 3.74$)$ & 0.163 & $2.03(1.02-4.01)$ & 0.043 \\
\hline \multicolumn{5}{|l|}{ Contralateral VA stenosis* } \\
\hline Hypoplasia & Ref & & Ref & \\
\hline$<70 \%$ & $0.91(0.30$ to 2.76$)$ & 0.868 & $0.95(0.33-2.74)$ & 0.920 \\
\hline$\geq 70 \%$ or occlusion & $3.32(0.96$ to 11.46$)$ & 0.058 & $3.23(0.98-10.65)$ & 0.055 \\
\hline \multicolumn{5}{|c|}{ Patients with severe anterior artery stenosis } \\
\hline \multicolumn{5}{|l|}{ NVAFV } \\
\hline 199.1-highest* & Ref & Ref & Ref & Ref \\
\hline $0-112.8$ & 14.03 (3.18 to 61.92$)$ & $<0.001$ & $11.99(3.10-46.3)$ & $<0.001$ \\
\hline $112.9-157.1$ & 1.55 (0.54 to 4.44$)$ & 0.416 & $1.60(0.58-4.38)$ & 0.363 \\
\hline $157.2-199$ & 1.04 (0.33 to 3.27$)$ & 0.950 & $1.91(0.70-5.20)$ & 0.207 \\
\hline \multicolumn{5}{|c|}{ Contralateral VA stenosis* } \\
\hline Hypoplasia & Ref & & Ref & \\
\hline$<70 \%$ & $1.47(0.27$ to 8.15$)$ & 0.658 & $1.43(0.29-6.96)$ & 0.662 \\
\hline$\geq 70 \%$ or occlusion & $2.77(0.45$ to 16.88$)$ & 0.269 & $2.72(0.50-14.93)$ & 0.249 \\
\hline \multicolumn{5}{|c|}{ Patients without severe anterior artery stenosis } \\
\hline \multicolumn{5}{|l|}{ NVAFV } \\
\hline 199.1-highest* & Ref & Ref & Ref & Ref \\
\hline $0-112.8$ & $2.76(0.85$ to 8.95$)$ & 0.090 & $1.83(0.65-5.17)$ & 0.257 \\
\hline $112.9-157.1$ & 2.08 (0.68 to 6.35$)$ & 0.197 & $1.38(0.52-3.68)$ & 0.516 \\
\hline $157.2-199$ & 2.34 (0.76 to 7.25$)$ & 0.141 & $1.98(0.74-5.33)$ & 0.174 \\
\hline \multicolumn{5}{|c|}{ Contralateral VA stenosis } \\
\hline Hypoplasia & Ref & & Ref & \\
\hline$<70 \%$ & $0.66(0.16$ to 2.94$)$ & 0.601 & $0.75(0.18-3.05)$ & 0.686 \\
\hline$\geq 70 \%$ or occlusion & 6.67 (0.91 to 49.04$)$ & 0.062 & $6.80(0.98-47.13)$ & 0.052 \\
\hline
\end{tabular}

*Adjust for age, sex, contralateral VA stenosis, anterior severe stenosis.

NVAFV, net vertebral artery flow volume; PCI, posterior circulation infarction; TIA, transient ischaemic stroke; VA, vertebral artery.

EVT for symptomatic VA stenosis was found either in the short-term or long-term on the occurrence of stroke or composite vascular outcome. Nevertheless, the author reported some findings that are worthy of note. First, all the trials enrolled patients with extracranial and intracranial VA stenosis as their target population. Existing data displayed a higher risk of recurrent ischaemia in intracranial VA stenosis than in extracranial stenosis. ${ }^{2}$ The treatment benefit may vary according to the stenosis location; however, none of the trials considered the stenosis site in analysing different endpoints. This was also proposed by Drazyk and Markus, ${ }^{20}$ with a review focusing on the stenosis site difference. Second, both the VIST and VAST clinical trials chose patients with $>50 \%$ symptomatic vertebrobasilar stenosis as their target population for endovascular intervention. ${ }^{79}$ In the VIST study, a high proportion of patients was included in the stent group who were found not to have stenosis. The effect of EVT might be masked under these circumstances since Guppy et al claimed that patients with $>70 \%$ stenosis of the vertebrobasilar system could have a profound flow volume change after angioplasty; however, for patients with only $50 \%$ stenosis, flow volume remained consistent after angioplasty. ${ }^{21}$ Stenting was used to augment the stenotic lumen to increase the flow volume and reduce the LAA incidence in PCI. Therefore, the greatest beneficiaries of stents were those with significantly reduced blood flow volume. In the ostial VA stenosis study, patients with bilateral VA pathology showed a significantly lower long-term risk of cerebral ischaemia recurrence after endovascular therapy. ${ }^{22}$ Third, EVT displayed an increasing periprocedural complication rate in the randomised trials. Hence, higher specificity for selecting high-risk flow compromised patients was much more important than higher 


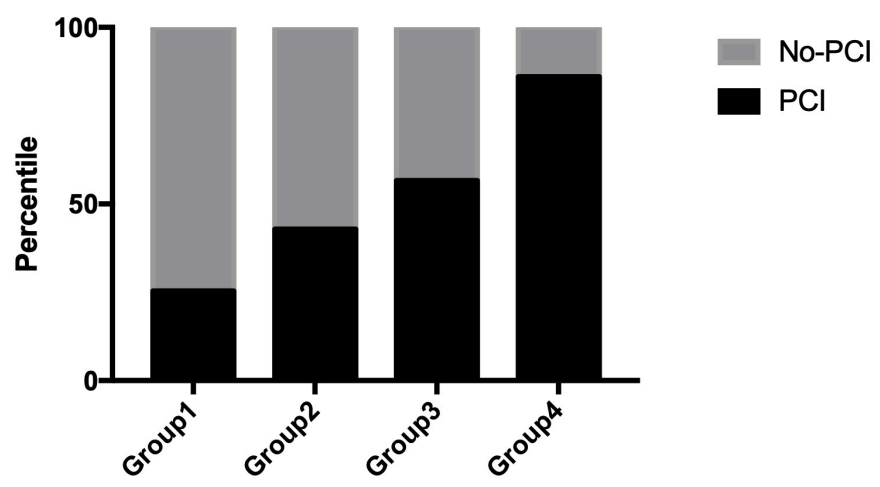

Figure 3 Distribution of $\mathrm{PCl}$ in each group divided by NVAFV and grades of contralateral VA stenosis. Group 1. CVAS(-) and H-NVAFV; group 2. CVAS(+) and H-NVAFV; group 3. CVAS(-) and L-NVAFV; group 4. CVAS(+) and L-NVAFV. CVAS, contralateral VA non-severe stenosis; $\mathrm{H}-$ NVAFV, higher net flow volume; L-NVAFV, lower NVAFV; PCI, posterior circulation infarction.

sensitivity to perform EVT. Highly specific stratification method is, therefore, more urgent and useful to identify the target population, which could prevent unnecessary interventions and complication risks in order to create a favourable balance between recurrent stroke events and periprocedural risk. In the present study, we demonstrated that reduced NVAFV was a strong predictor for PCI, especially via the LAA mechanism. Compared with stenosis grade, blood flow volume had a significant association with the prevalence of diseases. NVAFV would be a robust method to screen high-risk flow compromised patients in VA stenosis. A large multicentre cohort study should be conducted in the future to investigate the risks

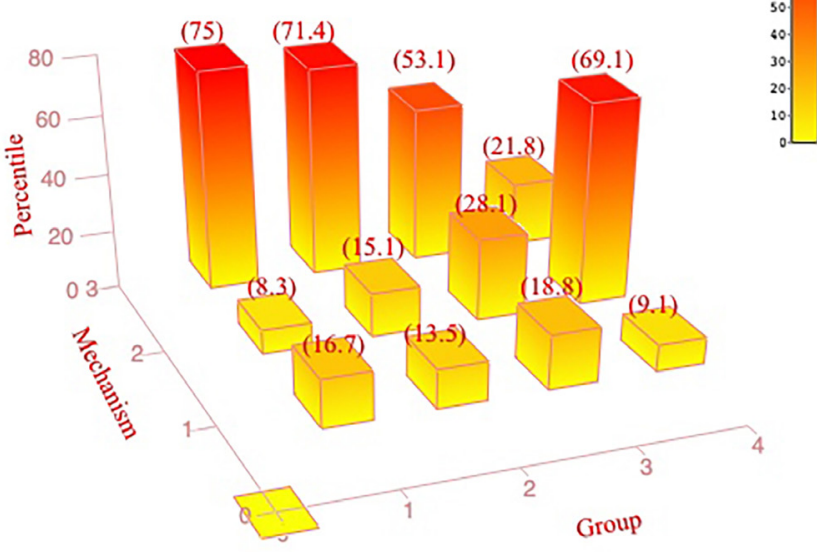

Figure 4 Distribution of $\mathrm{PCI}$ mechanisms in each group. Group 1. CVAS(-) and H-NVAFV; group 2. CVAS(+) and $\mathrm{H}-$ NVAFV; group 3. CVAS(-) and L-NVAFV; group 4. CVAS(+) and L-NVAFV. Mechanism classification: 1. Branch artery occlusive disease; 2. Large-artery atherosclerosis; 3. No PCl lesion. CVAS, contralateral VA non-severe stenosis; H-NVAFV, higher net flow volume; L-NVAFV lower NVAFV; PCI, posterior circulation infarction.

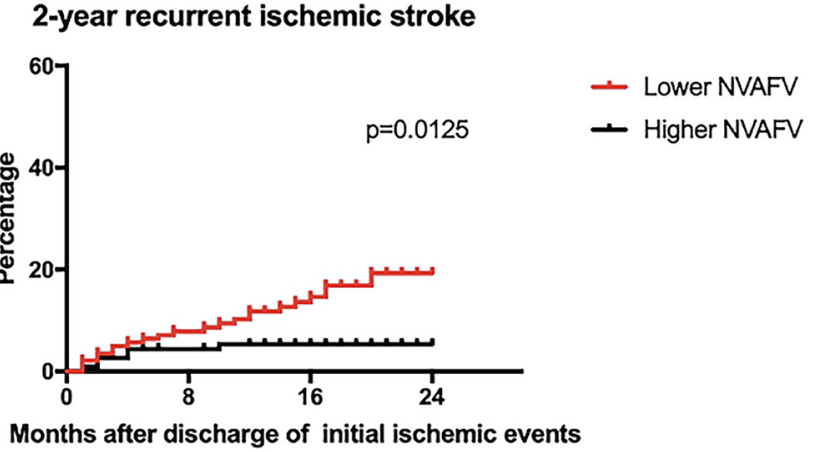

Figure 5 Recurrent ischaemic events between different level of net flow volume (NVAFV). Lower NVAFV group showed higher incidence of subsequent cerebral ischaemic events than higher NVAFV group.

and benefits of EVT in the higher risk population with NVAFV stratification.

Other investigators have measured distal blood flow volume in the VA using quantitative magnetic resonance angiography and have shown that reduced distal blood volume is associated with a higher risk of subsequent PCI during follow-up, ${ }^{23}$ whereas patients with normal distal flow volume have a low risk of subsequent stroke and do not require any intervention. This conclusion is consistent with our observations. Our follow-up examination demonstrated that lower NVAFV was associated with subsequent incidence of cerebral ischaemic events. However, because the positive events in our study were very few, we did not perform subgroup analysis. A part of the infarcted population may have had an anterior circulation infarction; this is a limitation of our study. We intend to continue this research to expand the sample size, thus allowing valid subgroup analysis.

Blood flow measurements in the internal carotid arteries and VA using CDU agreed with those measured using phase-contrast MRI, as reported previously. ${ }^{12} 24$ Since VA morphology is relatively uniform, NVAFV can be measured anywhere in the cervical VA. As flow volume measured with CDU was consistent that measured with phase-contrast MRI, we believe that NVAFV calculated by CDU could be an effective, convenient and accurate substitution.

In our study, severe anterior artery stenosis was predominant in the H-NVAFV group. Although few studies have investigated VA flow volume changes in patients with severe carotid artery disease, one study has reported an increase in NVAFV in patients with unilateral extracranial internal carotid artery occlusion. ${ }^{25}$ Our study findings support this idea and demonstrated the same results for anterior artery disease. We analysed this specific population separately using a multiple factor analysis because a H-NVAFV is not a physiological event. In this patient population, given that the OR of PCI with lower flow volume was 14.03 , as opposed to only 2.76 in patients without severe anterior arterial stenosis, we cannot 
overlook carotid stenosis in patients with lower NVAFV. A treatment strategy for the extracranial VA should be considered for patients with severe carotid artery stenosis and decompensation of the VA flow volume.

Our study also found that severe contralateral VA stenosis was a predictor for PCI; however, the influence was not as significant as that of NVAFV. It tended to correlate with PCI findings in patients without severe anterior artery stenosis; however, the relationship was not observed in patients with severe carotid artery stenosis. We found that the severe contralateral VA stenosis group exhibited an increased prevalence of PCI compared with the non-severe contralateral VA stenosis group. Under the same condition of severe contralateral VA stenosis, lower NVAFV was associated with a marked elevation in PCI compared with H-NVAFV. For strict and precise screening of the target population, NVAFV seemed to be more useful.

Some researchers believe that hypoplasia is a predictor for PCI or TIA. ${ }^{26-28}$ There is no consensus on the definition of hypoplasia, and a retrospective analysis has shown that VA of $<2 \mathrm{~mm}$ in diameter is predominant in patients with PCI. Another study that has defined VA hypoplasia as a diameter of $<2.5 \mathrm{~mm}$ has found that VA hypoplasia is frequent in patients with PCI. In our research group, we found that hypoplasia had no impact on PCI compared with severe stenosis. A partial reason for this is that the majority of our patient population was older than 65 years. In a previous study, the association between hypoplasia and PCI was found to be valid only in patients younger than 65 years. ${ }^{26}$ Another reason may be that we defined hypoplasia in our patient population as a diameter of $<2.5 \mathrm{~mm}$, which may not affect PCI.

Our study had several limitations. First, this was a retrospective study. Second, there were two types of populations in our non-PCI group, including patients with asymptomatic and anterior circulation infarction. Although we analysed the relationship between severe anterior artery stenosis and NVAFV separately, anterior circulation infarcts induced by small vessel disease may still influence the regional flow volume in the posterior circulation system. Third, NVAFV was calculated in patients with PCI, and we could only infer that there was a relationship between NVAFV and PCI, but we could not establish the cause. Finally, because of the small number of positive events noted during follow-up, we could not perform a precise subgroup analysis. As a next step, we plan to conduct a large size prospective cohort study to further define the relationship between flow volume and recurrent stroke events.

\section{CONCLUSION}

Our study demonstrated that NVAFV, as estimated by CDU, was associated with PCI and subsequent ischaemic events, especially in the LAA group, and we could identify a high-risk population for further revascularisation. In patients with simultaneous severe carotid artery disease, decompensation of the VA flow volume should trigger an alert for revascularisation.

Contributors QL collected the data, conducted data analysis and wrote the manuscript. YZ conceived the project and collected the data. YX revised the manuscript and commented on the data. JY offered important inputs on the results. $\mathrm{YH}$ was involved in the study design and manuscript revision. YH was responsible for the overall content as the guarantor. YH accepted full responsibility for the work, had access to the data, and controlled the decision to publish.

Funding The authors have not declared a specific grant for this research from any funding agency in the public, commercial or not-for-profit sectors.

Competing interests None declared.

Patient consent for publication Not applicable.

Ethics approval This retrospective cohort study was approved by the Ethics Committee of Capital Medical University Xuan Wu Hospital.

Provenance and peer review Not commissioned; externally peer reviewed. Data availability statement Data are available upon reasonable request. Not applicable.

Open access This is an open access article distributed in accordance with the Creative Commons Attribution Non Commercial (CC BY-NC 4.0) license, which permits others to distribute, remix, adapt, build upon this work non-commercially, and license their derivative works on different terms, provided the original work is properly cited, appropriate credit is given, any changes made indicated, and the use is non-commercial. See: http://creativecommons.org/licenses/by-nc/4.0/.

ORCID iD

Yang Hua http://orcid.org/0000-0002-6474-1065

\section{REFERENCES}

1 Markus HS, van der Worp HB, Rothwell PM. Posterior circulation ischaemic stroke and transient ischaemic attack: diagnosis, investigation, and secondary prevention. Lancet Neurol 2013;12:989-98.

2 Gulli G, Marquardt L, Rothwell PM, et al. Stroke risk after posterior circulation stroke/transient ischemic attack and its relationship to site of vertebrobasilar stenosis: pooled data analysis from prospective studies. Stroke 2013;44:598-604.

3 Qureshi Al, Ziai WC, Yahia AM, et al. Stroke-free survival and its determinants in patients with symptomatic vertebrobasilar stenosis: a multicenter study. Neurosurgery 2003;52:1033-9. discussion 9-40.

4 Stayman AN, Nogueira RG, Gupta R. A systematic review of stenting and angioplasty of symptomatic extracranial vertebral artery stenosis. Stroke 2011;42:2212-6.

5 Antoniou GA, Murray D, Georgiadis GS, et al. Percutaneous transluminal angioplasty and stenting in patients with proximal vertebral artery stenosis. J Vasc Surg 2012;55:1167-77.

6 Compter A, van der Worp HB, Schonewille WJ, et al. Vast: vertebral artery stenting trial. protocol for a randomised safety and feasibility trial. Trials 2008;9:65.

7 Compter A, van der Worp HB, Schonewille WJ, et al. Stenting versus medical treatment in patients with symptomatic vertebral artery stenosis: a randomised open-label phase 2 trial. Lancet Neurol 2015;14:606-14.

8 Markus HS, Larsson SC, Kuker W, et al. Stenting for symptomatic vertebral artery stenosis: the vertebral artery ischaemia stenting trial. Neurology 2017;89:1229-36.

9 Markus HS, Larsson SC, Dennis J, et al. Vertebral artery stenting to prevent recurrent stroke in symptomatic vertebral artery stenosis: the VIST RCT. Health Technol Assess 2019;23:1-30.

10 Caplan LR, Wityk RJ, Glass TA, et al. New England medical center posterior circulation registry. Ann Neurol 2004;56:389-98.

11 Seidel E, Eicke BM, Tettenborn B, et al. Reference values for vertebral artery flow volume by duplex sonography in young and elderly adults. Stroke 1999;30:2692-6.

12 Khan MA, Liu J, Tarumi T, et al. Measurement of cerebral blood flow using phase contrast magnetic resonance imaging and duplex ultrasonography. J Cereb Blood Flow Metab 2017;37:541-9.

13 Lee $\mathrm{H}-\mathrm{H}$, Huang L-K, Chang $\mathrm{H}-\mathrm{J}$, et al. Association between net vertebral artery flow volume and Non-AF stroke: a retrospective 2year analysis. Front Neurol 2018;9:1198.

14 Kernan WN, Ovbiagele B, Black HR, et al. Guidelines for the prevention of stroke in patients with stroke and transient ischemic attack: a guideline for healthcare professionals from the 
American heart Association/American stroke association. Stroke 2014;45:2160-236.

15 Hua Y, Meng X-F, Jia L-Y, et al. Color Doppler imaging evaluation of proximal vertebral artery stenosis. AJR Am J Roentgenol 2009;193:1434-8.

16 North American Symptomatic Carotid Endarterectomy Trial Collaborators, Barnett HJM, Taylor DW, et al. Beneficial effect of carotid endarterectomy in symptomatic patients with high-grade carotid stenosis. N Engl J Med 1991;325:445-53.

17 Caplan L, Chung C-S, Wityk R, et al. New England medical center posterior circulation stroke registry: I. Methods, data base, distribution of brain lesions, stroke mechanisms, and outcomes. $J$ Clin Neurol 2005;1:14-30.

18 Adams HP, Bendixen BH, Kappelle LJ, et al. Classification of subtype of acute ischemic stroke. Definitions for use in a multicenter clinical trial. TOAST. trial of ORG 10172 in acute stroke treatment. Stroke 1993;24:35-41.

19 Lattanzi S, Brigo F, Di Napoli M, et al. Endovascular treatment of symptomatic vertebral artery stenosis: a systematic review and meta-analysis. J Neurol Sci 2018;391:48-53.

20 Drazyk AM, Markus HS. Recent advances in the management of symptomatic vertebral artery stenosis. Curr Opin Neurol 2018;31:1-7.
21 Guppy KH, Charbel FT, Corsten LA, et al. Hemodynamic evaluation of basilar and vertebral artery angioplasty. Neurosurgery 2002;51:327-34. discussion 33-4.

22 Karameshev A, Schroth G, Mordasini P, et al. Long-term outcome of symptomatic severe ostial vertebral artery stenosis (OVAS). Neuroradiology 2010;52:371-9.

23 Amin-Hanjani S, Pandey DK, Rose-Finnell L, et al. Effect of hemodynamics on stroke risk in symptomatic atherosclerotic vertebrobasilar occlusive disease. JAMA Neurol 2016;73:178-85

24 Oktar SO, Yücel C, Karaosmanoglu D, et al. Blood-flow volume quantification in internal carotid and vertebral arteries: comparison of 3 different ultrasound techniques with phase-contrast MR imaging. AJNR Am J Neuroradiol 2006;27:363-9.

25 Nicolau C, Gilabert R, García A, et al. Effect of internal carotid artery occlusion on vertebral artery blood flow: a duplex ultrasonographic evaluation. J Ultrasound Med 2001;20:105-11.

26 Gaigalaite V, Vilimas A, Ozeraitiene V, et al. Association between vertebral artery hypoplasia and posterior circulation stroke. BMC Neurol 2016;16:118.

27 Park J-H, Kim J-M, Roh J-K. Hypoplastic vertebral artery: frequency and associations with ischaemic stroke Territory. J Neurol Neurosurg Psychiatry 2007;78:954-8.

28 Perren F, Poglia D, Landis T, et al. Vertebral artery hypoplasia: a predisposing factor for posterior circulation stroke? Neurology 2007;68:65-7. 